\title{
Seventh International Congress of Human Genetics Berlin
}

\section{Clinical cytogenetics}

In his introduction to the Clinical Cytogenetics Symposium, Albert de la Chapelle stressed how valuable traditional microscopical cytogenetics has been in directing molecular biologists to the relevant chromosomal segments, so that linkage of RFLPs to important monogenic conditions could be established. He mentioned as examples the role of constitutional structural chromosomal rearrangements for the mapping of the Duchenne muscular dystrophy locus to Xp21, cystic fibrosis to $7 \mathrm{q} 31$, and retinoblastoma to $13 q 14$. These examples were well chosen, among other things because one of the most exciting take home messages from this Conference was the news that the next step from linkage to locus may have been taken as regards retinoblastoma, and also for a portion of the Duchenne/Becker muscular dystrophy gene defect. Whatever the true nature of the latter, it will certainly be a relief for everybody involved in prenatal diagnosis in Duchenne muscular dystrophy families, including clinical cytogeneticists, that the condition may now be adequately diagnosed in the majority of chromosomally male fetuses and at an early gestational age.

In contrast to the rapid molecular diagnostic developments of monogenic conditions, there was little new on offer for the clinical cytogeneticist as regards the microscopical diagnosis of chromosomal disorders. As pointed out by Ferguson-Smith in the workshop on chromosome sorting, automated karyotyping is not yet a feasible option, as the equipment required is prohibitively expensive. Large numbers of long and nicely banded chromosomes were paraded by many speakers, and the potential of in situ hybridisation of DNA probes by ${ }^{3} \mathrm{H}$-thymidine incorporation was elegantly demonstrated by Mattei (who being of French extraction is committed to $\mathrm{R}$ banding). The problem is, however, that this technology is too time consuming to be of any direct practical value in a busy diagnostic Cytogenetic Unit. There was no indication of any relevant development in non-radioactive in situ

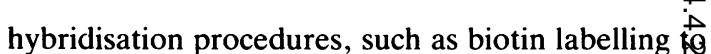
aid the identification of small deletions in syndrome such as Prader-Willi, Giedion-Langer, DiGeorge, and Miller-Dieker, which even by current extended banding, and in the best of hands, are troublesome and not very reliable. For some time clinic ? cytogeneticists have had the hope that some such technology would prove helpful as an adjuvant traditional cytogenetics, and a cocktail of probes from any specific chromosome should presumab? allow the identification of numerical abnormalities even in interphase nuclei.

In her review on $\mathrm{X}$ linked mental retardatio Margareta Mikkelson pointed out that the fragile syndrome has now been shown to be much mor complex than previously envisaged. Cytogenetic diagnosis and genetic counselling in these families has become a real headache. She stressed particular that in the grandparental generation about $20 \%$ of asymptomatic males, although neith mentally retarded nor carrying the $X$ markeonevertheless transmit the condition to their offspring. In addition, $30 \%$ of 'heterozygous' females may be mentally retarded, sometimes very severely. On piece of good news from Tommerup was that $\mathrm{a}$ reliable diagnosis of fetal fragile $X$ might be obtained after culture of chorionic villus samples for one to two weeks in vitro.

One of the Symposia in Berlin, and also $2 \widetilde{P}$ International Conference in Vienna the week after were dedicated to the Rett syndrome. Since 1983 there has been increasing interest in this syndrome also initially believed to be $\mathrm{X}$ linked. To date about 1200 girls have been diagnosed as having the syndrome. A normal sex ratio in the sibships make it unlikely that this is an $\mathrm{X}$ linked condition with lethality in males. Most of the cases have bee 'sporadic' but there is some indication of a heredita predisposition. Two pairs of full sisters and two se of affected monozygotic twins have been describe 8 The syndrome has also been seen in two half sisters 
who shared the same mother but had different fathers.

Extensive cytogenetic investigations by Wahlstrom in Sweden raised initial hopes of an association with a fragile site at $\mathrm{Xp} 21$, but this has not been confirmed by further analyses. With increasing awareness of the clinical features of this neurodegenerative syndrome many more cases are likely to be diagnosed in the near future, and hopefully the enigma of its origin and sex preference may be unravelled.

Maj Hultén Regional Cytogenetics Laboratory, East Birmingham Hospital, Bordesley Green East, Birmingham B9 5ST.

\section{Ethical practices}

Two recent meetings have highlighted some of the ethical issues which are important to both the 'old' and the 'new' genetics.

At the International Congress of Human Genetics, one of the best organised sessions was the symposium 'Ethical and legal issues in applied human genetics' chaired by the peripatetic ethicist, John C Fletcher (NIH). Good clear presentations, kept strictly to time, allowed full discussion and made a welcome relief at a congress notable for its vast numbers and prolixity. The workshop was opened by Fletcher and his colleague Dorothy Wertz, who described a crosscultural study begun in 1984. Four 'dominant moral approaches' may, according to Fletcher, be evolving as shown by the results of a survey: 643 medical geneticists from 17 countries replied from the 1020 sent the questionnaire. The four 'dominant moral approaches' are (1) parental (that is, consultand) autonomy; (2) the obligation to reduce or prevent the suffering caused by genetic disease on the condition that parental autonomy is respected; (3) a duty to provide full disclosure of test results (including colleague disagreements); and (4) a voluntary rather than mandatory approach to genetic screening except for newborns when treatment is available. The ethical principles which appear to be widely accepted across all countries are Autonomy, Beneficience, Non-maleficience, and Justice (or equity).

Of the specific problems put to medical geneticists in the questionnaire, those that created greatest ethical conflict included confidentiality of a diagnosis of Huntington's chorea versus duties to relatives at risk, disclosure of an $\mathrm{XY}$ genotype in a female, and prenatal diagnosis for sex selection (with no $X$ linked disease involved). It was fascinating in the discussion to hear that the practice in Canada was to reject the use of prenatal diagnosis for sex selection, although it had appeared from the questionnaire returns that a majority in Canada (and the United States and Hungary) would either perform it or refer to someone else who might. According to Fletcher, individual medical geneticists would admit views in an anonymous questionnaire which they would not implement or even disclose in public. It also emerged that the private sector might be a factor in the development of such fringe activities in North America. In other cultures sex selection is anything but 'fringe', but these views were not discussed. (Reprints of the full paper can be obtained from Dr Fletcher, Holding 10, Room 2C-202 NIH Bethesda, Maryland 20892, USA.)

Bernard Dickens (Toronto) followed Fletcher by discussing 'Comparative law and legislation on eugenic sterilisation and selective abortion'. This was a valuable summary of present and past laws, providing incidentally a wide ranging historical review of eugenics and the crimes that had been committed in its name in the United States and Germany. Together with Arno Motulsky's references to German eugenics in a splendid presidential address, these provided, most appropriately in Berlin, a sort of genetic catharsis.

The third presentation in the symposium was given by Helga Kuhse (Monash, Australia) who discussed many of the now familiar arguments about embryo research in a practical and even pragmatic way. Since the theme was that early human embryos do not have a 'right to life', this was followed, not unexpectedly, by a heated discussion including the reading of a prepared statement from the audience.

RODNEY HARRIS Department of Medical Genetics, St Mary's Hospital, Hathersage Road, Manchester M13 OJH.

\section{Royal College of Physicians Conference on Medical Ethics, 23 October 1986}

This was much less specialised, dealing with a very wide range of ethical issues including euthanasia, 